\title{
Efficacy of Ceftazidime-Avibactam in the
}

\section{Treatment of Carbapenem-Resistant Klebsiella pneumoniae Infection After Kidney Transplantation}

\author{
Fei Zhang $\mathbb{D}^{1-3, *}$ \\ Jinbiao Zhong ${ }^{1-3, *}$ \\ Handong Ding ${ }^{1-3, *}$ \\ Guiyi Liao $\mathbb{D}^{1-3}$
}

'Department of Urology, The First Affiliated Hospital of Anhui Medical University, Hefei City, Anhui Province, People's Republic of China; ${ }^{2}$ Institute of Urology, The First Affiliated Hospital of Anhui Medical University, Hefei City, Anhui Province, People's Republic of China; ${ }^{3}$ Anhui Province Key Laboratory of Genitourinary Diseases, Anhui Medical University, Hefei City, Anhui Province, People's Republic of China

*These authors contributed equally to this work
Correspondence: Guiyi Liao

Department of Urology, The First Affiliated Hospital of Anhui Medical University, No. 218 Jixi Road, Shushan District, Hefei City, Anhui Province,

People's Republic of China

Tel +86-I58569I5237

Email liaoguiyi@ahmu.edu.cn
Purpose: The clinical efficacy of ceftazidime-avibactam (CAZ-AVI) in treating carbapenem-resistant Klebsiella pneumoniae (CRKP)-infected recipients after kidney transplantation (KT) has not been well evaluated. We aimed to assess its efficacy in a single-center cohort of KT recipients infected with CRKP.

Materials and Methods: We retrospectively observed KT recipients diagnosed with CRKP infection from June 2019 to July 2021. The primary outcome was 30-day mortality and secondary outcomes were 14-day clinical cure and 14-day microbiological cure. Logistic regression analysis was used to evaluate the relationship between CAZ-AVI treatment and prognosis.

Results: A total of 54 CRKP-infected KT recipients were recorded in this study. Twenty-two recipients received CAZ-AVI and 32 received other antibiotic regimens. Recipients in both groups had similar baseline characteristics, with the most common site of infection being surgical site infections $(n=27 ; 50.0 \%)$ and bloodstream infections $(n=23 ; 42.6 \%)$. Recipients treated with CAZ-AVI had significantly lower 30-day mortality $(3 / 22$ vs $14 / 32, \mathrm{P}=0.019)$, significantly higher 14 -day clinical cure $(18 / 22$ vs $17 / 32, \mathrm{P}=0.030)$ and 14-day microbiological cure $(19 / 22$ vs $15 / 32, \mathrm{P}=0.003)$ compared with recipients receiving other treatment regimens. Kaplan-Meier survival curves for 30-day mortality confirmed the findings (logrank=0.014). In a multivariate logistic regression model, receiving CAZ-AVI was found to be an independent protective factor for 30-day mortality (odds ratio $=0.148,95 \%$ confidence interval, 0.027-0.800; $\mathrm{P}=0.026$ ). No significant side effects were recorded.

Conclusion: CAZ-AVI may be more valuable than other antibiotic regimens for the treatment of CRKP infection after kidney transplantation, and further large randomized controlled trials are needed to assess its efficacy.

Keywords: ceftazidime-avibactam, carbapenem-resistant Klebsiella pneumoniae, kidney transplantation, infections

\section{Introduction}

Infection is a major cause of morbidity and mortality in solid organ transplant (SOT) recipients. ${ }^{1}$ SOT recipients are prone to multidrug-resistant bacterial infections due to factors such as the use of immunosuppressive agents, prolonged ICU stay and more invasive procedures, which are important factors that affect the postoperative survival. ${ }^{2}$ Among them, carbapenem-resistant Klebsiella pneumoniae (CRKP) has become one of the most lethal pathogenic infections following kidney transplantation $(\mathrm{KT}){ }^{3}$ The case fatality rate of CRKP infection in SOT recipients has been reported to be up to $43 \%$, with a mortality rate 
approximately 3-5 times higher than those not infected with CRKP. $^{3,4}$ The percentage of CRKP isolates in China significantly increased from $4.9 \%$ in 2013 to $10.9 \%$ in 2019 obtained from the CHINET surveillance of bacterial resistance. Such strains are resistant to most current antibiotics, including $\beta$-lactams, fluoroquinolones, and carbapenems. ${ }^{5}$ Although polymyxin and tigecycline remain the most effective options for the treatment of CRKP in vitro, these drugs have limited clinical use due to toxicity or inefficiency, particularly in kidney transplant recipients, many drugs for CRKP (such as polymyxin or amikacin) have high nephrotoxicity, and the use of these drugs is often limited given the protection of kidney allograft function. ${ }^{6,7}$ There are extremely limited clinical treatment options for CRKP infection after $\mathrm{KT}$, and the marketing of CAZ-AVI provides a new treatment option for CRKP infection.

CAZ-AVI is a combination of a third-generation cephalosporin (ceftazidime) and a novel $\beta$-lactamase inhibitor (avibactam), marketed in the United States in February 2015. It is the first antibiotic approved by the Food and Drug Administration for the treatment of carbapenem-resistant Enterobacteriaceae (CRE) infections, approved for the treatment of complicated intra-abdominal infections, complicated urinary tract infections, hospital-acquired, ventilator-associated pneumonia, and infections caused by aerobic gram-negative bacteria in adult patients with limited treatment options. ${ }^{8,9}$ Avibactam prevents the hydrolysis of ceftazidime by many enzymes, including Ambler class A, C and D $\beta$-lactamases (e.g, extendedSpectrum $\beta$-Lactamases [ESBLs], AmpC, K. pneumoniae carbapenemases [KPCs] and OXA-48), thereby restoring activity against bacteria that produce these enzymes. CAZ-AVI is ineffective against class B metalloenzymes (IMP, VIM, NDM) due to the lack of active site serine residues in class B enzymes. ${ }^{10,11}$ Previous studies have reported that CAZ-AVI has higher clinical success and survival rates compared with other antibiotic regimens for the treatment of infections caused by CRKP. ${ }^{12,13}$ However, assessment of CAZ-AVI efficacy in the treatment of CRKP specifically in KT recipients has not been reported. Therefore, the aim of this study was to compare the efficacy of CAZ-AVI and other antibiotic regimens in the KT population treated for CRKP infection.

\section{Materials and Methods}

\section{Study Design and Patient Sample}

We conducted a retrospective study of CRKP-infected recipients from June 2019 to July 2021, in which culture results of all CRKP strains were screened. Adult CRKPinfected recipients ( $\geq 18$ years of age) receiving CAZ-AVI for $\geq 72 \mathrm{~h}$ and other antibiotic-treated CRKP-infected recipients were included in the study, while recipients with colonized or contaminated culture results were excluded. We compared CRKP-infected recipients treated with CAZAVI and those treated with other antibiotics during this period, and only the first course was considered if the patient received more than one CAZ-AVI treatment. Baseline characteristics of CRKP-infected recipients were recorded, clinical, microbiological, and therapeutic characteristics were collected, and data on outcomes 30 days after the onset of infection were obtained. All data in this study were extracted from the electronic medical record information system in our hospital. The primary outcome was 30-day mortality, and secondary outcomes were 14day clinical cure and 14-day microbiological cure. Severity at the onset of infection was measured using the Acute Physiology and Chronic Health Evaluation II (APACHE II $^{14}$ and the Sequential Organ Failure Assessment (SOFA). ${ }^{15}$ All kidneys were donated by relatives or deceased citizens, and all kidneys were donated voluntarily with written informed consent, which was conducted in accordance with the Declaration of Istanbul. This study was approved by our institutional Ethics Review Committee and was conducted in accordance with the principles of the Declaration of Helsinki.

\section{Definition}

The standards used to define and classify infections in our study were those proposed by the Centers for Disease Control and Prevention. ${ }^{16}$ There were four main types of infection, which were pneumonia (including ventilatorassociated infections), surgical site infections (SSIs), bloodstream infections (BSIs) (including catheterassociated infections) and urinary tract infections (UTIs). The date of infection onset was defined as the date of collection when the specimen was first cultured positive. Thirty-day mortality was defined as all-cause death 30 days after the onset of infection. Fourteen-day clinical failure and 14-day microbiological failure were defined as events occurring within 14 days from the date of treatment initiation with the study drug. Clinical failure was defined as meeting any of the following criteria: I, death; II, persistent symptoms or signs of infection; III, relapse. Microbiological cure was defined as a negative subsequent sample culture (in patients without a repeat sample, the presence of a clinical cure was also considered 
a microbiological cure). Infection relapse was defined as the presence of a second microbiologically documented CRKP infection in a patient whose initial infection was classified as a clinical cure. Monotherapy was defined as a treatment regimen using a single in vitro active drug. Combination therapy was defined as the addition of other antimicrobial agents with in vitro activity or resistance $(\geq 72 \mathrm{~h}$ ) against clinical isolates. Salvage therapy was defined as antibiotic therapy given after clinical or microbiological failure of the first-line regimen, or when the previous treatment could not be continued because of severe side effects. ${ }^{17}$

\section{Immunosuppressive Regimen and Use of Antibiotics}

All the selected recipients received triple immunosuppression (tacrolimus or cyclosporin A, prednisone, and mycophenolate mofetil), and some received additional anti-human thymocyte immunoglobulin. The standard doses of CAZAVI were $2.0 \mathrm{~g}$ ceftazidime and $0.5 \mathrm{~g}$ avibactam intravenously every 8 hours for more than 2 hours each time. Dosage and administration were adjusted according to kidney function, as per the manufacturer's recommendations. ${ }^{18}$ Tigecycline was administered at a dose of $100 \mathrm{mg}$ twice daily (after a loading dose of $200 \mathrm{mg}$ ).

\section{Microbiology}

Susceptibility testing was performed using the VITEK-2 system (Biomerieux, Marcy-l 'Etoile, France) and disc diffusion method. Minimum inhibitory concentrations were interpreted according to breakpoints established by the Clinical and Laboratory Standards Institute. ${ }^{19}$ CRKP was defined as insensitivity to at least one carbapenem, with a minimum inhibitory concentration $\geq 2 \mathrm{mg} / \mathrm{mL}$ for ertapenem and $\geq 4 \mathrm{mg} / \mathrm{mL}$ for imipenem or meropenem. ${ }^{19}$

\section{Statistical Analysis}

Statistical analysis was performed using SPSS software (version 25.0; SPSS Inc., Chicago, IL). Continuous variables with normal distribution were described by means and standard deviation, otherwise data were represented by median and interquartile range (IQR). The independentsample $\mathrm{t}$ tests and Mann-Whitney $U$-test were used to compare normal and non-normal continuous variables, respectively. Categorical variables were represented by frequency and percentage. Chi-square or Fischer exact tests were used for comparison of categorical variables between groups, as appropriate. A multivariate logistic regression model was used to identify risk factors for 30day mortality. Variables emerging from univariate analysis with $\mathrm{P}$ values of $<0.05$ were included in the multivariate model in a backward stepwise manner. Kaplan-Meier analysis and Log rank test were used to evaluate differences in the 30-day survival curves of CRKP-infected recipients treated with or without CAZ-AVI. $\mathrm{P}<0.05$ was considered statistically significant.

\section{Results}

According to the medical records, a total of 58 recipients were diagnosed with CRKP infection between June 2019 and July 2021 in our hospital. Two CRKP-infected recipients who received CAZ-AVI for $<72 \mathrm{~h}$ and two CRKP recipients who had lost follow-up data were excluded from the study, leaving a total of 54 recipients enrolled in the study. Twenty-two received CAZ-AVI, and the 32 recipients in the comparative group received other antibiotics. There were 47 recipients with subsequent culture collection, 20 of which were still culture positive.

\section{Baseline Characteristics}

The most common etiology was glomerulonephritis in both groups. The mean age of the two groups was similar: $37.2 \pm 9.8$ years in the CAZ-AVI group and $41.1 \pm 10.0$ years in the comparative group $(\mathrm{P}=0.164)$. The SOFA and APACHE II scores were $4.2 \pm 2.1$ and $9.7 \pm 3.2$, respectively, in the CAZ-AVI group and $4.3 \pm 1.9$ and $10.6 \pm 3.0$, respectively, in the comparative group $(\mathrm{P}=0.967$ and 0.299 , respectively). Table 1 lists the remaining demographic characteristics and baseline characteristics of the two groups of recipients and there were no significant differences in the baseline data between the two treatments groups.

\section{Antibiotic Administration}

Table 1 also summarizes the characteristics of therapy in CRKP infection recipients. The median time from onset of infection to initiation of CAZ-AVI or other antibiotics was similar in the two groups: 2.0 (2.0-9.0) versus 3.0 (2.0-3.0) days $(\mathrm{P}=0.369)$, respectively. The mean durations of treatment were also similar: (10.7 \pm 4.4) versus $(10.4 \pm 4.8)$ days $(\mathrm{P}=0.821)$, respectively. In the CAZ-AVI group, $72.7 \%$ (16/22) and 27.3\% (6/ 22) of recipients received either single therapy or combination therapy, respectively, with seven recipients receiving salvage therapy of CAZ-AVI and 15 
Table I Baseline Characteristics and Outcomes of Recipients with CRKP Infections Who Received Ceftazidime/Avibactam Compared with Other Antibiotic Treatment Regimens (Comparative Group)

\begin{tabular}{|c|c|c|c|}
\hline \multirow[t]{2}{*}{ Characteristics } & \multirow{2}{*}{$\begin{array}{c}\text { CAZ-AVI Group } \\
\mathrm{N}=\mathbf{2 2}(\%)\end{array}$} & \multirow{2}{*}{$\begin{array}{c}\text { Comparative Group } \\
\qquad \mathrm{N}=32(\%)\end{array}$} & \multirow[t]{2}{*}{$P$ value } \\
\hline & & & \\
\hline Deceased donors & $17(77.3)$ & $27(84.4)$ & 0.509 \\
\hline Age(years), mean $\pm S D$ & $37.2 \pm 9.8$ & $41.1 \pm 10.0$ & 0.164 \\
\hline Sex, male n (\%) & $13(59.1)$ & $19(59.4)$ & 0.983 \\
\hline BMI $(\mathrm{kg} / \mathrm{m} 2)$, mean $\pm S D$ & $21.3 \pm 3.0$ & $22.2 \pm 2.0$ & 0.194 \\
\hline Diabetes mellitus & $6(27.3)$ & $9(28.1)$ & 0.945 \\
\hline \multicolumn{4}{|l|}{ Etiology of kidney failure, n (\%) } \\
\hline HTA & $2(9.1)$ & $4(12.5)$ & 0.695 \\
\hline DM & $2(9.1)$ & $4(12.5)$ & 0.695 \\
\hline Glomerulonephritis & $16(72.7)$ & $19(59.4)$ & 0.313 \\
\hline Others & $2(9.1)$ & $5(15.6)$ & 0.482 \\
\hline \multicolumn{4}{|l|}{ Type of dialysis n (\%) } \\
\hline Hematodialysis & $15(68.2)$ & $19(59.4)$ & 0.510 \\
\hline Peritoneal dialysis & $7(31.8)$ & $13(40.6)$ & 0.510 \\
\hline ATG induction $\mathrm{n}(\%)$ & $12(54.5)$ & $19(59.4)$ & 0.724 \\
\hline \multicolumn{4}{|l|}{ Types of infections } \\
\hline BSIs & II (50.0) & $12(37.5)$ & 0.361 \\
\hline UTIs & $5(22.7)$ & $10(31.3)$ & 0.492 \\
\hline Pneumonia & $9(40.9)$ & $12(37.5)$ & 0.801 \\
\hline SSls & $14(63.6)$ & $13(40.6)$ & 0.097 \\
\hline SOFA at infection onset, mean \pm SD & $4.2 \pm 2.1$ & $4.3 \pm 1.9$ & 0.967 \\
\hline APACHE II at infection onset, mean \pm SD & $9.7 \pm 3.2$ & $10.6 \pm 3.0$ & 0.299 \\
\hline Source control & $6(27.3)$ & $9(28.1)$ & 0.945 \\
\hline Time from positive culture to study drug initiation(days), median (IQR) & $2.0(2.0-9.0)$ & $3.0(2.0-3.0)$ & 0.369 \\
\hline Study drug within $48 \mathrm{~h}, \mathrm{n}(\%)$ & II (50.0) & $15(46.9)$ & 0.821 \\
\hline Duration of study drug(days), mean $\pm S D$ & $10.7 \pm 4.4$ & $10.4 \pm 4.8$ & 0.821 \\
\hline Duration of infection after transplantation, mean $\pm S D$ & $11.5 \pm 6.2$ & $11.5 \pm 5.4$ & 0.977 \\
\hline Graft loss & I (4.5) & $5(15.6)$ & 0.207 \\
\hline Relapse, n (\%) & $2(9.1)$ & $7(21.9)$ & 0.215 \\
\hline 14-day clinical cure, n (\%) & $18(81.8)$ & $17(53.1)$ & 0.030 \\
\hline 14-day microbiological cure, n (\%) & $19(86.4)$ & $15(46.9)$ & 0.003 \\
\hline 30-day mortality, n (\%) & $3(13.6)$ & $14(43.8)$ & 0.019 \\
\hline
\end{tabular}

Notes: The variable marked in bold indicates that the difference between the two groups is statistically significant $(P<0.05)$.

Abbreviations: CRKP, carbapenem-resistant Klebsiella pneumoniae; CAZ-AVI, ceftazidime/avibactam; BMI, body mass index; HTA, hypertension; DM, diabetes mellitus; ATG, anti-human thymocyte immunoglobulin; BSIs, bloodstream infections; UTIs, urinary tract infections; SSIs, surgical site infections; SOFA, Sequential Organ Failure Assessment; APACHE II, Acute Physiologic Assessment and Chronic Health Evaluation II.

recipients receiving initial therapy of CAZ-AVI. In the comparative group, $62.5 \%(20 / 32)$ and $37.5 \%(12 / 32)$ of the recipients received treatment alone and in combination, respectively. Details of the specific use of antibiotics in the two groups are detailed in Table 2. Susceptibility of CRKP isolates is shown in Table 3. 
Table 2 Frequency of Antibiotic Regimen Used for Treatment of CRKP Infections in Kidney Transplantation

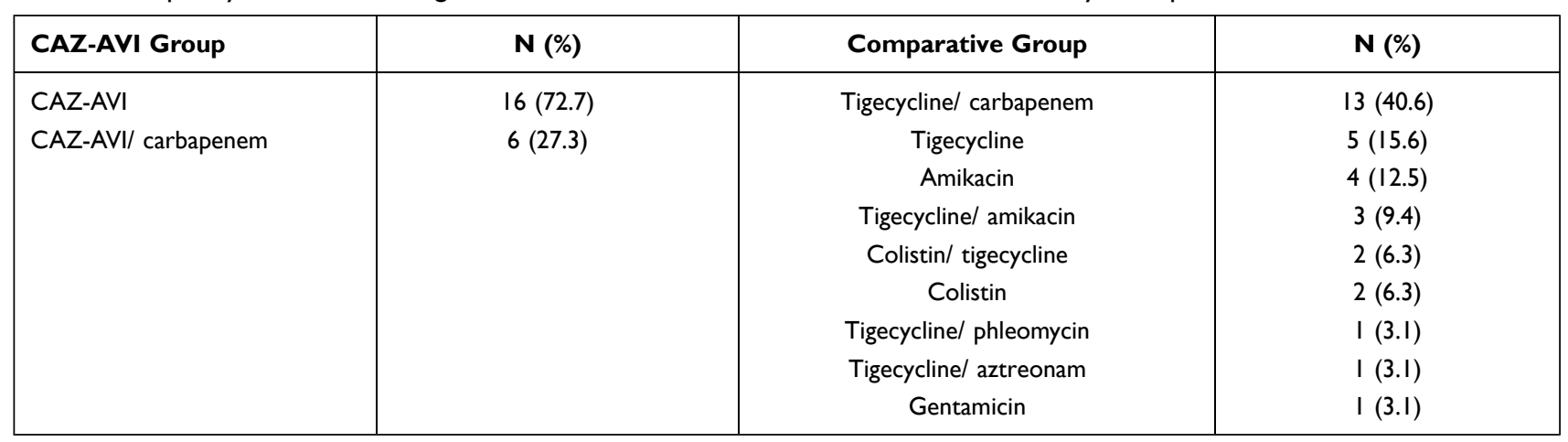

\section{Outcomes}

The 30-day mortality of the CAZ-AVI group was significantly lower than that of the comparative group $(13.6 \% \mathrm{vs}$ $43.8 \% ; \mathrm{P}=0.019)$. The 30 -day mortality rate was $3 / 16$ $(18.8 \%)$ and $0 / 6(0.0 \%)$ in recipients receiving CAZ-AVI alone and in combination with carbapenems, respectively. Fourteen-day clinical cure was observed in 18/22 (81.8\%) recipients in the CAZ-AVI group and in $17 / 32(53.1 \%)$ recipients in the comparative group $(\mathrm{P}=0.030)$, while 14 day microbiological cure was noted in 19/22 (86.4\%) recipients in the CAZ-AVI group and in $15 / 32(46.9 \%)$ recipients in the comparative group $(\mathrm{P}=0.003)$. Infection relapse was observed in $2 / 22(9.1 \%)$ recipients in the CAZ-AVI group and in $7 / 32(21.9 \%)$ recipients in the comparative group ( $\mathrm{P}=0.215)$. Graft loss occurred in one and five recipients in the CAZ-AVI and comparative groups, respectively, and graft loss rates were similar between the two groups $(4.5 \%$ vs $15.6 \%, \mathrm{P}=0.203)$. During the whole study, no adverse reactions related to CAZ-AVI were found.

Table 3 Antimicrobial Susceptibility of Isolates from Recipients with CRKP Infections

\begin{tabular}{|l|c|c|}
\hline Antibiotic & $\begin{array}{c}\text { Number of Isolates } \\
\text { Tested(N) }\end{array}$ & $\begin{array}{c}\text { Susceptible } \\
\text { (\%) }\end{array}$ \\
\hline Ceftazidime & 54 & 0.0 \\
Levofloxacin & 54 & 9.3 \\
Gentamycin & 53 & 13.2 \\
Imipenem & 54 & 0.0 \\
Meropenem & 54 & 0.0 \\
Amikacin & 50 & 24.0 \\
Polymyxin & 52 & 96.2 \\
Tigecycline & 54 & 98.1 \\
Ceftazidime- & 40 & 100.0 \\
Avibatam & & \\
\hline
\end{tabular}

\section{Relationship Between CAZ-AVI} Treatment and Prognosis

The survival curves for the two treatment groups are shown in Figure 1, with 30-day survival of the CAZ-AVI recipients significantly higher than that of recipients treated with other antibiotic regimens (log-rank $=0.014)$. Univariate and multivariate analyses of 30-day survival and death are shown in Table 4. Multivariate logistic regression analysis showed that CAZ-AVI treatment was an independent predictor of 30-day survival (odds ratio [OR] $=0.148,95 \%$ confidence interval [CI] 0.027-0.800; $\mathrm{P}=0.026)$. The model obtained had an area under the receiver operating characteristic curve of 0.851 (as shown in Figure 2). The timing of appropriate antibiotic treatment initiation did not differ between survivors and non-survivors $(2[2,4.5]$ vs $3[2,4]$ days, $\mathrm{P}=0.0 .311)$. There was also no significant difference in the duration of antibiotic treatment $(10.3 \pm 4.2$ vs $11.2 \pm 5.4$ days, $\mathrm{P}=0.497$ ).

\section{Discussion}

To our knowledge, this is the first study to compare the clinical outcomes of CAZ-AVI versus other antibiotic regimens for the treatment of CRKP infection after KT specifically in a KT recipient population. Our study showed that in CRKP-infected KT recipients, CAZ-AVI was associated with significantly lower 30-day mortality and significantly higher 14-day clinical and microbiological cures compared with other antibiotics. We also found that the use of an antibiotic regimen containing CAZ-AVI was an independent protective factor for 30 day mortality. In our study, clinical and microbiological efficacy was assessed 14 days after initiation of treatment, rather than the usual 30 days in previous studies, ${ }^{20}$ considering that it may be more accurate to assess the efficacy of antibiotic therapy early. 


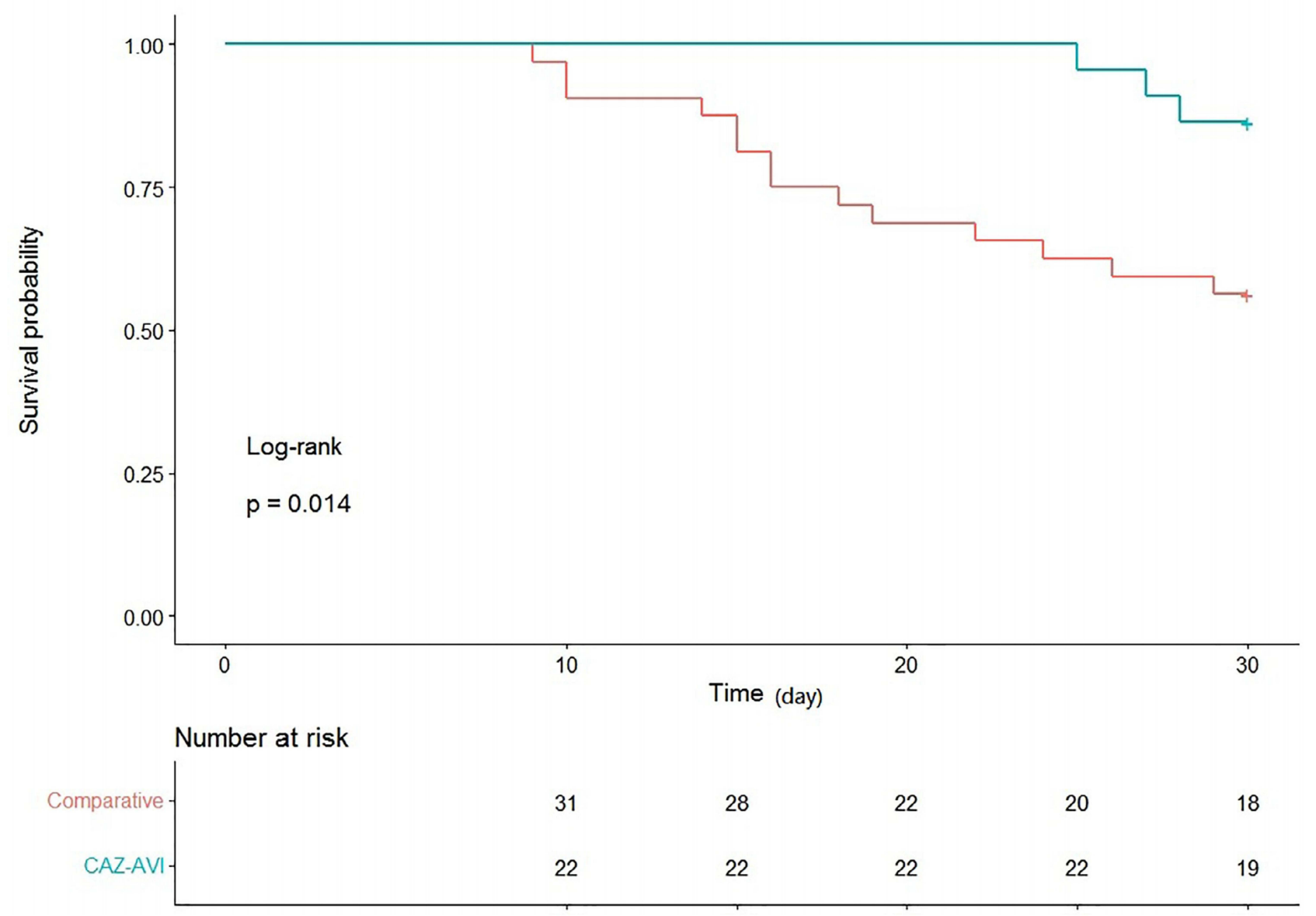

Figure I The survival curves for the two treatment groups between CAZ-AVI and comparative group.

CAZ-AVI has emerged as a promising therapy for CRKP infection in several clinical studies, ${ }^{21,22}$ In a retrospective observational study comparing 42 patients with CRKP infection treated with CAZ-AVI and 48 patients treated with other antibiotics, it was shown that both 14-day clinical cure and 14day microbiological cure were significantly improved in the CAZ-AVI group compared with the comparative group (66.7\% vs $50 \%, \mathrm{P}=0.046 ; 73.8 \%$ vs $68.7 \%, \mathrm{P}=0.034)$, and the 30-day mortality rate was $19.0 \%$ in the CAZ-AVI group, ${ }^{12}$ which was similar to the 30 -day mortality of $13.6 \%$ in the CAZ-AVI group in our study. Tumbarello et al also reported a similar 30-day mortality rate of $25 \%$ in 577 patients with CRKP infection treated with CAZ-AVI. ${ }^{22}$ Shields et al compared 13 patients with CRKP infection who received CAZ-AVI with 96 patients receiving other antibiotics. The clinical cure rate and 30-day survival rate in CAZ-AVI group were significantly higher than those without CAZ-AVI. ${ }^{13}$ In another study, 38 CRE patients treated with CAZ-AVI were compared with 99 CRE patients treated with polymyxin, demonstrating that the 30-day mortality after initiation of treatment was significantly lower in the CAZ-AVI group ( $9 \%$ vs $32 \% ; \mathrm{P}=0.001) .{ }^{23}$ Two of $22(9.1 \%)$ recipients in the CAZAVI group in this study developed infection relapse, which was similar to the relapse rate of $23 \%$ in a study of 37 CRE-infected patients treated with CAZ-AVI. ${ }^{20}$ Furthermore, our multivariate analysis found that the use of a treatment regimen containing CAZ-AVI was an independent predictor of 30-day survival, which is consistent with previous findings. ${ }^{21}$

Previous studies have reported that CAZ-AVI showed a greater benefit in patients with higher disease severity. In a retrospective study in Greece, CAZ-AVI was found to be more effective than other antibiotics in treating patients with CRE infection in an intensive care unit population with greater severity of illness or mechanical ventilation. ${ }^{24} \mathrm{Gu}$ et $\mathrm{al}^{12}$ also found CAZ-AVI to be more valuable in treating severe CRKP infections than in treating mild CRKP infections. Although there was no significant difference in disease severity between the CAZ-AVI group and the comparative group in our study, possibly related to the small sample size, it is necessary to further evaluate the efficacy of CAZ-AVI in the treatment of 
Table 4 Univariate and Multivariate Analysis of 30-Day Mortality of Recipients with CRKP Infection After Kidney Transplantation

\begin{tabular}{|c|c|c|c|c|}
\hline \multirow[t]{2}{*}{ Characteristics } & Survivors & $\begin{array}{c}\text { Non- } \\
\text { Survivors }\end{array}$ & \multirow[t]{2}{*}{$P$ value } & \multirow[t]{2}{*}{$\begin{array}{l}\text { Multivariate } P \text { value }(\mathrm{OR}, 95 \% \\
\mathrm{CI})\end{array}$} \\
\hline & $\mathbf{N}=37(\%)$ & $N=\mid 7(\%)$ & & \\
\hline Deceased donors & $31(83.8)$ & $13(76.5)$ & 0.523 & \\
\hline Age(years), mean $\pm S D$ & $37.4 \pm 10.4$ & $44.1 \pm 7.4$ & 0.028 & \\
\hline Sex, male n (\%) & $2 \mathrm{I}(56.8)$ & II (64.7) & 0.582 & \\
\hline BMI $(\mathrm{kg} / \mathrm{m} 2)$, mean $\pm S D$ & $21.9 \pm 2.4$ & $21.5 \pm 2.8$ & 0.567 & \\
\hline Diabetes & $9(24.3)$ & $6(35.3)$ & 0.406 & \\
\hline \multicolumn{5}{|l|}{ Etiology of kidney failure, $n$ (\%) } \\
\hline HTA & $5(13.5)$ & $\mathrm{I}(5.9)$ & 0.420 & \\
\hline DM & $4(10.8)$ & $2(11.8)$ & 0.918 & \\
\hline Glomerulonephritis & $25(67.6)$ & $10(58.8)$ & 0.533 & \\
\hline Others & $3(8.1)$ & $4(23.5)$ & 0.133 & \\
\hline \multicolumn{5}{|l|}{ Type of dialysis n (\%) } \\
\hline Hematodialysis & $22(59.5)$ & $12(70.6)$ & 0.434 & \\
\hline Peritoneal dialysis & $15(40.5)$ & $5(29.4)$ & 0.434 & \\
\hline ATG induction $\mathrm{n}(\%)$ & $21(56.8)$ & $10(58.8)$ & 0.887 & \\
\hline \multicolumn{5}{|l|}{ Types of infections } \\
\hline BSIs & $15(40.5)$ & $8(47.1)$ & 0.653 & \\
\hline UTIs & $\mathrm{II}(29.7)$ & $4(23.5)$ & 0.637 & \\
\hline Pneumonia & $13(35.1)$ & $8(47.1)$ & 0.406 & \\
\hline SSls & $20(54.1)$ & $7(41.2)$ & 0.381 & \\
\hline SOFA at infection onset, mean $\pm S D$ & $3.8 \pm 2.1$ & $5.3 \pm 1.0$ & 0.012 & \\
\hline APACHE II at infection onset, mean \pm SD & $9.4 \pm 3.0$ & $12.2 \pm 2.3$ & 0.004 & \\
\hline Source control & $7(18.9)$ & 8(47.1) & 0.037 & \\
\hline $\begin{array}{l}\text { Time from positive culture to study drug initiation(days), median } \\
\text { (IQR) }\end{array}$ & $2(2-4.5)$ & $3(2-4)$ & 0.311 & \\
\hline Study drug within $48 \mathrm{~h}, \mathrm{n}(\%)$ & $20(54.1)$ & $6(35.3)$ & 0.204 & \\
\hline Duration of study drug(days), mean $\pm S D$ & $10.3 \pm 4.2$ & $11.2 \pm 5.4$ & 0.497 & \\
\hline Duration of infection after transplantation, mean $\pm S D$ & $10.6 \pm 5.2$ & $13.4 \pm 6.3$ & 0.099 & \\
\hline Relapse, n (\%) & $4(10.8)$ & $5(29.4)$ & 0.100 & \\
\hline CAZ-AVI contamin, $n(\%)$ & $19(51.4)$ & $3(17.6)$ & 0.026 & $0.026(0.148,0.027-0.800)$ \\
\hline
\end{tabular}

Notes: Bold values indicated that these variables were significant in univariate and multivariate analysis $(P<0.05)$.

Abbreviations: CRKP, carbapenem-resistant Klebsiella pneumoniae; CAZ-AVI, ceftazidime/avibactam; BMI, body mass index; HTA, hypertension; DM, diabetes mellitus; ATG, anti-human thymocyte immunoglobulin; BSIs, bloodstream infections; UTIs, urinary tract infections; SSls, surgical site infections; SOFA, Sequential Organ Failure Assessment; APACHE II, Acute Physiologic Assessment and Chronic Health Evaluation II.

CRKP infection subgroups in the KT population. The proportion of SSIs type was higher in recipients in the CAZ-AVI group than in the comparative group, and although not statistically significant, SSIs could reduce the bacterial load by removing the infected lesion through debridement and drainage compared with infection at other sites, which may be a confounding factor between the two groups, which needs more cases to be further explored.

Currently, the effect of combination therapy with CAZAVI on CRE infection remains controversial. In our study, recipients treated with CAZ-AVI alone had higher 30-day mortality compared with recipients treated with CAZ-AVI 


\section{ROC Curve}

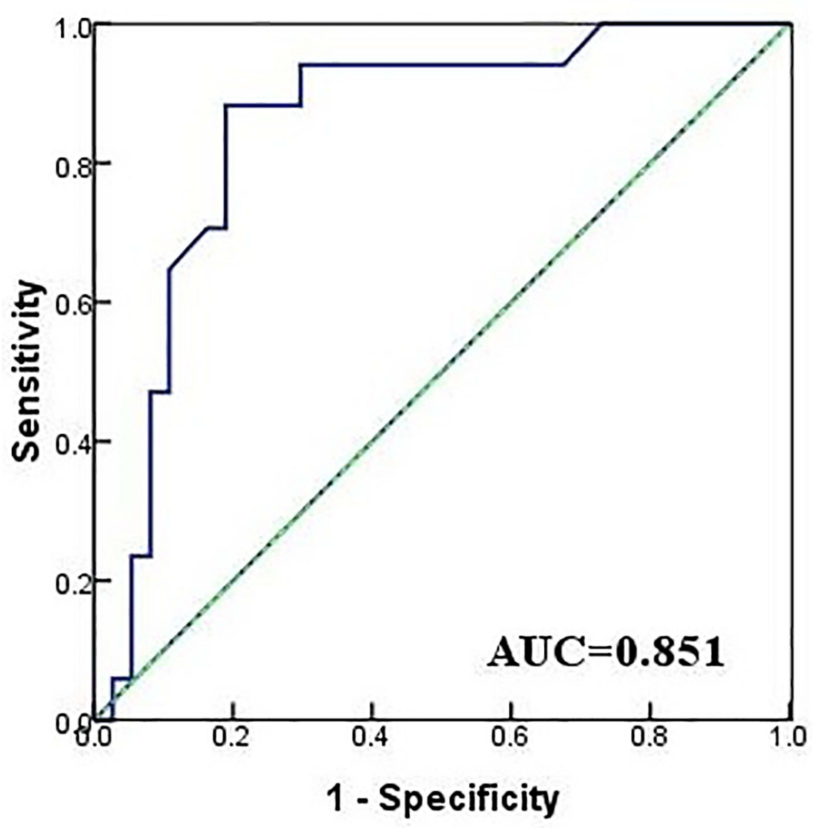

Figure 2 Area under the receiver operating characteristic curve for this model.

combination therapy, which was in contrast to the findings of Gu et al ${ }^{12}$ who found that patients treated with CAZ-AVI alone had a lower 30-day mortality. This discrepancy may be caused by a proportion of CAZ-AVI recipients receiving salvage therapy in our study, and the other important cause may be the low number of recipients in CAZ-AVI/carbapenem group. In a study of bloodstream infections caused by $\mathrm{CRKP}^{25}$ early initiation of appropriate antibiotic therapy was associated with lower 30-day mortality. A study by Zheng et $\mathrm{al}^{26}$ found that CAZ-AVI combined with in vitro insensitive drugs significantly reduced the 30-day mortality in CRKP-infected patients compared with CAZ-AVI alone, which is consistent with our findings. In a model of Galleria Mellonella larvae infected with CRKP, CAZ-AVI combined with carbapenem significantly improved the survival rate and inhibited the development of drug resistance compared with CAZ-AVI alone. ${ }^{27}$ However, a recent meta-analysis showed that combination therapy with CAZ-AVI was not associated with improved clinical outcomes, ${ }^{28}$ requiring larger randomized controlled trials to address this issue. CAZ-AVI can also be used as salvage therapy in patients with CRKP infection, with similarly good clinical and microbiological cure rates. ${ }^{17}$ One study has reported that the 30-day mortality rate of 138 patients with CRKP infection who received CAZ-AVI as salvage therapy was approximately $34.1 \%$, consistent with the 30 -day mortality in our study. ${ }^{21}$
The emergence of CAZ-AVI-resistant strains during CAZ-AVI treatment has been reported. Shields et $a^{20}$ described the emergence of three CAZ-AVI-resistant strains in 37 CRE patients treated with CAZ-AVI, and in another retrospective study of 47 CRKP-infected recipients treated with CAZ-AVI, ${ }^{29}$ it was also found that six patients developed CAZ-AVI resistance during treatment. This may be related to metallo- $\beta$-lactamase production, bla-KPC point mutation and high expression of KPC. ${ }^{30,31}$ Although none of the CRKP isolates in this study showed CAZ-AVI drugresistant strains, this may be related to the lack of representative microbiological data in our study, and CAZ-AVI should be included in the standard drug sensitivity test.

Our study had several limitations. First, this was a retrospective observational study, treatment was not randomly assigned, and there might be some confounding factors. Second, the number of patients included in this study was small, and our study has insufficient statistical power to detect significant differences in clinical and microbiological efficacy, making it difficult to generalize our conclusions to other patients or other conditions outside our center. Larger randomized controlled trials are required to resolve the problem. Third, we do not have data regarding carbapenemase genes, which are related to drug resistance mechanisms.

\section{Conclusion}

The present study shows that CAZ-AVI is superior to other antibiotic regimens in the treatment of CRKP infections in KT populations regardless of the gene resistance of CRKP. Although this study has limitations and is small in size, it is the largest comparative study in the KT population, to date, and shows that CAZ-AVI is a promising antibiotic for the treatment of CRKP-infected recipients with limited treatment options after KT. However, further studies are needed to determine the efficacy of CAZ-AVI in the treatment of CRKP infections after KT.

\section{Abbreviations}

CAZ-AVI, ceftazidime-avibactam; CRKP, carbapenemresistant Klebsiella pneumoniae; KT, kidney transplantation; SOT, solid organ transplant; CRE, carbapenems-resistant Enterobacteriaceae.

\section{Ethical Approval}

The study was approved by the Ethics Committee of The First Affiliated Hospital of Anhui Medical University (Approval Number Quick-PJ 2021-13-17). In the retrospective cohort, the requirement of informed consent 
from study participants was waived because of the retrospective and anonymized nature of this study.

\section{Acknowledgments}

We thank Gillian Campbell, PhD, from Liwen Bianji (Edanz) (www.liwenbianji.cn/), for editing the English text of a draft of this manuscript.

\section{Author Contributions}

All authors made a significant contribution to the work reported, whether that is in the conception, study design, execution, acquisition of data, analysis and interpretation, or in all these areas; took part in drafting, revising or critically reviewing the article; gave final approval of the version to be published; have agreed on the journal to which the article has been submitted; and agree to be accountable for all aspects of the work.

\section{Funding}

This work received funding from the Natural Science Foundation of Anhui Province (Grant No. 1508085SMH226).

\section{Disclosure}

The authors report no conflicts of interest in this work.

\section{References}

1. Kim SI. Bacterial infection after liver transplantation. World J Gastroenterol. 2014;20(20):6211-6220. doi:10.3748/wjg.v20.i20.6211

2. Aguado JM, Silva JT, Fernandez-Ruiz M, et al. Management of multidrug resistant Gram-negative bacilli infections in solid organ transplant recipients: SET/GESITRA-SEIMC/REIPI recommendations. Transplant Rev. 2018;32(1):36-57. doi:10.1016/j.trre.2017.07.001

3. Xu L, Sun X, Ma X. Systematic review and meta-analysis of mortality of patients infected with carbapenem-resistant Klebsiella pneumoniae. Ann Clin Microbiol Antimicrob. 2017;16(1):18. doi:10.1186/s12941017-0191-3

4. Pouch SM, Kubin CJ, Satlin MJ, et al. Epidemiology and outcomes of carbapenem-resistant Klebsiella pneumoniae bacteriuria in kidney transplant recipients. Transpl Infect Dis. 2015;17(6):800-809. doi:10.1111/tid.12450

5. Meletis G. Carbapenem resistance: overview of the problem and future perspectives. Ther Adv Infect Dis. 2016;3(1):15-21. doi:10.1177/ 2049936115621709

6. Livermore DM, Warner M, Mushtaq S, Doumith M, Zhang J, Woodford N. What remains against carbapenem-resistant Enterobacteriaceae? Evaluation of chloramphenicol, ciprofloxacin, colistin, fosfomycin, minocycline, nitrofurantoin, temocillin and tigecycline. Int J Antimicrob Agents. 2011;37(5):415-419. doi:10.1016/j.jjantimicag.2011.01.012

7. Chiu SK, Wu TL, Chuang YC, et al. National surveillance study on carbapenem non-susceptible Klebsiella pneumoniae in Taiwan: the emergence and rapid dissemination of KPC-2 carbapenemase. PLoS One. 2013;8(7):e69428. doi:10.1371/journal.pone.00 69428
8. Karaiskos I, Lagou S, Pontikis K, Rapti V, Poulakou G. The "Old" and the "New" antibiotics for MDR Gram-negative pathogens: for whom, when, and how. Front Public Health. 2019;7:151. doi:10.3389/fpubh.2019.00151

9. Das S, Li J, Riccobene TJ, Carrothers TJ, Newell P. Dose selection and validation for ceftazidime-avibactam in adults with complicated intra-abdominal infections, complicated urinary tract infections, and nosocomial pneumonia. Antimicrob Agents Chemother. 2019;63(4): e02187-18. doi:10.1128/AAC.02187-18

10. Doi Y. Treatment options for carbapenem-resistant Gram-negative bacterial infections. Clin Infect Dis. 2019;69(Suppl 7):S565-S575. doi:10.1093/cid/ciz830

11. Zhanel GG, Lawson CD, Adam H, et al. Ceftazidime-avibactam: a novel cephalosporin/ $\beta$-lactamase inhibitor combination. Drugs. 2013;73(2):159-177. doi:10.1007/s40265-013-0013-7

12. Gu J, Xu J, Zuo TT, Chen YB. Ceftazidime-avibactam in the treatment of infections from carbapenem-resistant Klebsiella pneumoniae: ceftazidime-avibactam against CR-KP infections. $J$ Glob Antimicrob Resist. 2021;26:20-25. doi:10.1016/j.jgar.2021.04.022

13. Shields RK, Nguyen MH, Chen L, et al. Ceftazidime-avibactam is superior to other treatment regimens against carbapenem-resistant Klebsiella pneumoniae bacteremia. Antimicrob Agents Chemother. 2017;61(8):e00883-17. doi:10.1128/AAC.00883-17

14. LeGall JR, Loirat P, Alpcrovitch A. APACHE II_ a severity of disease classification system. Crit Care Med. 1986;14(8):754-755. doi:10.1097/00003246-198608000-00027

15. Vincent JL, Moreno R, Takala J, et al. The SOFA (Sepsis-related Organ Failure Assessment) score to describe organ dysfunction/failure. Intensive Care Med. 1996;22(7):707-710. doi:10.1007/BF017 09751

16. Horan TC, Andrus M, Dudeck MA. CDC/NHSN surveillance definition of health care-associated infection and criteria for specific types of infections in the acute care setting. Am J Infect Control. 2008;36 (5):309-332. doi:10.1016/j.ajic.2008.03.002

17. Temkin E, Torre-Cisneros J, Beovic B, et al. Ceftazidime-avibactam as salvage therapy for infections caused by carbapenem-resistant organisms. Antimicrob Agents Chemother. 2017;61(2):Feb. doi:10.1128/AAC.01964-16

18. Rodriguez-Nunez O, Ripa M, Morata L, et al. Evaluation of ceftazidime/avibactam for serious infections due to multidrug-resistant and extensively drug-resistant Pseudomonas aeruginosa. $J$ Glob Antimicrob Resist. 2018;15:136-139. doi:10.1016/j.jgar.2018.07.010

19. Clinical and Laboratory Standards Institute. M100-S28. Performance standards for antimicrobial susceptibility testing: 28th informational supplement. Wayne, PA: Clinical and Laboratory Standards Institute; 2018.

20. Shields RK, Potoski BA, Haidar G, et al. Clinical outcomes, drug toxicity, and emergence of ceftazidime-avibactam resistance among patients treated for carbapenem-resistant Enterobacteriaceae infections. Clin Infect Dis. 2016;63(12):1615-1618. doi:10.1093/ $\mathrm{cid} / \mathrm{ciw} 636$

21. Tumbarello M, Trecarichi EM, Corona A, et al. Efficacy of ceftazidimeavibactam salvage therapy in patients with infections caused by Klebsiella pneumoniae carbapenemase-producing K. pneumoniae. Clin Infect Dis. 2019;68(3):355-364. doi:10.1093/cid/ciy492/5035217

22. Tumbarello M, Raffaelli F, Giannella M, et al. Ceftazidime-avibactam use for Klebsiella pneumoniae carbapenemase-producing K. pneumoniae infections: a retrospective observational multicenter study. Clin Infect Dis. 2021;73(9):1664-1676. doi:10.1093/cid/ciab176

23. van Duin D, Lok JJ, Earley M, et al. Colistin versus ceftazidime-avibactam in the treatment of infections due to carbapenem-resistant Enterobacteriaceae. Clin Infect Dis. 2018;66 (2):163-171. doi:10.1093/cid/cix783 
24. Tsolaki V, Mantzarlis K, Mpakalis A, et al. Ceftazidime-avibactam to treat life-threatening infections by carbapenem-resistant pathogens in critically ill mechanically ventilated patients. Antimicrob Agents Chemother. 2020;64(3). doi:10.1128/AAC.02320-19

25. Falcone M, Bassetti M, Tiseo G, et al. Time to appropriate antibiotic therapy is a predictor of outcome in patients with bloodstream infection caused by KPC-producing Klebsiella pneumoniae. Crit Care. 2020;24(1):29. doi:10.1186/s13054-020-2742-9

26. Zheng G, Zhang J, Wang B, et al. Ceftazidime-avibactam in combination with in vitro non-susceptible antimicrobials versus ceftazidime-avibactam in monotherapy in critically ill patients with carbapenem-resistant Klebsiella pneumoniae infection: a retrospective cohort study. Infect Dis Ther. 2021;10 (3):1699-1713. doi:10.1007/s40121-021-00479-7

27. Gottig S, Frank D, Mungo E, et al. Emergence of ceftazidime/avibactam resistance in KPC-3-producing Klebsiella pneumoniae in vivo. $J$ Antimicrob Chemother. 2019;74(11):3211-3216. doi:10.1093/jac/ $\mathrm{dkz} 330$
28. Fiore M, Alfieri A, Di Franco S, et al. Ceftazidime-avibactam combination therapy compared to ceftazidime-avibactam monotherapy for the treatment of severe infections due to carbapenem-resistant pathogens: a systematic review and network meta-analysis. Antibiotics. 2020;9(7):388. doi:10.3390/antibiotics9070388

29. Caston JJ, Gallo M, Garcia M, et al. Ceftazidime-avibactam in the treatment of infections caused by KPC-producing Klebsiella pneumoniae: factors associated with clinical efficacy in a single-center cohort. Int J Antimicrob Agents. 2020;56(3):106075. doi:10.1016/j. ijantimicag.2020.106075

30. Zhang P, Shi Q, Hu H, et al. Emergence of ceftazidime/avibactam resistance in carbapenem-resistant Klebsiella pneumoniae in China. Clin Microbiol Infect. 2020;26(1):124e1-124e4. doi:10.1016/j.cmi.201 9.08.020

31. Li D, Liao W, Huang $\mathrm{HH}$, et al. Emergence of hypervirulent ceftazidime/avibactam-resistant Klebsiella pneumoniae isolates in a Chinese tertiary hospital. Infect Drug Resist. 2020;13:2673-2680. doi:10.2147/IDR.S257477

\section{Publish your work in this journal}

Infection and Drug Resistance is an international, peer-reviewed openaccess journal that focuses on the optimal treatment of infection (bacterial, fungal and viral) and the development and institution of preventive strategies to minimize the development and spread of resistance. The journal is specifically concerned with the epidemiology of antibiotic resistance and the mechanisms of resistance development and diffusion in both hospitals and the community. The manuscript management system is completely online and includes a very quick and fair peerreview system, which is all easy to use. Visit http://www.dovepress.com/ testimonials.php to read real quotes from published authors. 